\title{
Pareto-Optimal Reinsurance Policies under TrTVaR Risk Measure
}

\author{
Yadong Li, Ying Fang \\ School of Mathematics and Statistics, Shandong Normal University, Jinan, China \\ Email: 17865814720@163.com, fangying319@163.com
}

How to cite this paper: Li, Y. D., \& Fang, Y. (2021). Pareto-Optimal Reinsurance Policies under TrTVaR Risk Measure. Journal of Financial Risk Management, 10, 260-273. https://doi.org/10.4236/jfrm.2021.103015

Received: August 8, 2021

Accepted: August 27, 2021

Published: August 30, 2021

Copyright $\odot 2021$ by author(s) and Scientific Research Publishing Inc. This work is licensed under the Creative Commons Attribution International License (CC BY 4.0).

http://creativecommons.org/licenses/by/4.0/

\begin{abstract}
In this paper, we consider the problem of optimal reinsurance design, when the risk is measured by TrTVaR risk measure. We study optimal reinsurance models from the perspectives of both insurers and reinsurers. To reduce ex-post moral hazard, we assume that reinsurance contracts satisfy the principle of indemnity and the incentive-compatible constraint. When the losses of an insurer and a reinsurer are both measured by TrTVaR risk measures, we obtain the explicit forms of the Pareto-optimal reinsurance contracts under the expected value premium principle and TVaR premium principle, respectively.
\end{abstract}

\section{Keywords}

TrTVaR Risk Measure, Pareto-Optimal Reinsurance, TVaR Premium

Principle, The Expected Value Premium Principle

\section{Introduction}

In the original insurance transaction, the policyholder transfers the loss to the insurer by signing an insurance contract. Reinsurance refers to the act of the insurer transferring some of the risks assumed by the insurer to other insurers on the basis of the original insurance contract by signing a reinsurance contract. A reinsurance policy is a contract, according to which part of the risk of an insurance company (the ceding company) is transferred to another insurance company (the reinsurance company), in exchange for receiving a premium. The basis of reinsurance is primary insurance, it is due to the need of primary insurers to disperse risks that reinsurance is generated. Reinsurance is an important tool for the insurer to manage risks, through reinsurance, the insurer can mitigate the underwriting risk, thereby facilitating more effective risk management.

To the best of our knowledge, (Borch, 1960) and (Arrow, 1963) studied the 
optimal reinsurance problems of variance minimization and expected utility maximization from the perspective of an insurer. The results have been extended to a risk-measure-based framework later. The problem of optimal reinsurance design has been studied by using risk measures, due to their development and application in finance and insurance. For instance, in a framework where the ceding company's risk is measured by Value-at-Risk (VaR) and Conditional Tail Expectation (CTE), with the expected value premium principle as the risk premium, (Cai \& Tan, 2007) found the optimal retention levels in a stop-loss reinsurance. Later, in the same framework, (Cai, Tan, Weng, \& Zhang, 2008) proved that depending on the risk measure level of confidence, the optimal reinsurance for an insurer, which minimizes the VaR and CTE of the total risk of the insurer, can be in the form of a stop-loss reinsurance or a quota-share reinsurance or a changeloss reinsurance under the expected value principle and among the increasing convex ceded loss functions. In (Bernard \& Tian, 2009) also, the authors have considered optimal risk management strategies of an insurance company subject to regulatory constraints when the risk is measured by VaR and CVaR (Conditional Value-at-Risk). Subsequently, researchers have tried to extend the optimal reinsurance design problem to larger families of risk measures and risk premiums. For instance, (Cheung, 2010) and (Chi \& Tan, 2013) extended the problem by using a family of general risk premiums; in these two papers the risk of the ceding company is measured either by VaR or CTE. On the other hand, (Cheung, Sung, Yam, \& Yung, 2014) have extended the problem by using general law-invariant convex risk measures, whereas the risk premium is considered to be the expected value premium principle.

As far as we know, a reinsurance contract involves the interests of both the insurer and the reinsurer. In a reinsurance strategy, Pareto-optimality can maximize the interests of both parties, that is one party makes its own interests better while not harming the interests of the other party. Pareto-optimal reinsurance has been well studied under various settings in insurance and risk management. For instance, (Jiang, Ren, Zitikis, \& Tang, 2017) proved that the optimal reinsurance strategy is a Pareto-optimal reinsurance policy and gave optimal reinsurance strategies using the geometric method. (Cai, Liu, \& Wang, 2017) and (Jiang, Hong, \& Ren, 2018) studied the Pareto-optimality of reinsurance arrangements under general model settings and obtained the explicit forms of the Pareto-optimal reinsurance contracts under the TVaR measure and the expected value premium principle. By geometric approach, (Fang, Wang, Liu, \& Li, 2019) studied Pareto-optimal reinsurance policies under general premium principles and gave the explicit parameters of the optimal ceded loss functions under Dutch premium principle and Wang's premium principle. (Jiang, Hong, \& Ren, 2021) studied the Pareto-optimal reinsurance strategy by considering the two optimization criteria of maximizing expected utility and minimizing risk measurement.

In this paper, we use a more robust and sensitive risk measure, namely the Truncated Tail Value-at-Risk (TrTVaR). Considering the interests of both the insurer and the reinsurer, the explicit solutions of Pareto-optimality are given. The 
rest of the paper is organized as follows: in the Second Section, we introduce some definitions and model formulation, including the definition and properties of TrTVaR risk measure, then we show that the form of Pareto-optimal reinsurance policies can be determined by minimizing linear combinations of the cedent's and the reinsurer's risks; in the Third Section, we determine the optimal reinsurance by giving a concrete form of Pareto-optimal reinsurance strategy under expected value premium principle and $\mathrm{TVaR}$ premium principle, respectively; in the Fourth Section, we give a summary description and point out directions and problems of the follow-up research.

\section{Model Setup}

Let a random variable $X$ denote the loss faced by the insurer without reinsurance with support $[0, M]$ where $M \leq \infty$. We assume that $X$ is defined in probability space $(\Omega, \mathbb{F}, \mathbb{P})$ with cumulative distribution function (c.d.f.) $F_{X}(x)=P\{X \leq x\}$, and survival function $S_{X}(x)=1-F_{X}(x)$. A reinsurance policy is to separate the loss $X$ into $R(X)$ and $I_{R}(X)$ with $X=I_{R}(X)+R(X)$, so that $R(X)$ satisfying $0 \leq R(X) \leq X$, is the loss ceded to a reinsurer, while $I_{R}(X)$ is the loss retained by the insurer. Therefore, $f(x)$ and $I_{R}(x)$ are the insurer's ceded loss function and the retained loss function. Then the insurer's total liability $M_{R}$ contains two parts: one is the retained loss risk $X-R(X)$ and the other is the reinsurance premium $P_{R}$, that is $M_{R}=X-R(X)+P_{R}$. The reinsurer's total liability $N_{R}$ becomes $N_{R}=R(X)-P_{R}$.

In the insurance and other financial industry, risk measures have been widely used for quantifying risk and setting regulatory capital. (Cont, Deguest, \& Scandalo, 2010) studied the robustness and sensitivity analysis of risk measures, they proposed an alternative for the TVaR. Formally, the definitions of some common risk measures are given below:

Definition 1. For a random variable $X$, VaR is defined as:

$$
\operatorname{VaR}_{\alpha}(X)=\inf \{x \in R: P(X \leq x) \geq \alpha\}
$$

where $0<\alpha<1$ represents a confidence level of the loss variable $X$.

Definition 2. For a random variable $X$, TVaR is defined as:

$$
\begin{aligned}
\operatorname{TVaR}_{\alpha}(X) & =\frac{1}{1-\alpha} \int_{\alpha}^{1} \operatorname{VaR}_{s}(X) \mathrm{d} s \\
& =\operatorname{VaR}_{\alpha}(X)+\frac{1}{1-\alpha} E\left(X-\operatorname{VaR}_{\alpha}(X)\right)_{+},
\end{aligned}
$$

where $0<\alpha<1$ represents a confidence level of the loss variable $X$.

Actually, VaR is a quantile and the VaR of a generic loss variable $X$ at a confidence level $\alpha, \operatorname{VaR}_{\alpha}(X)$, represents the minimum amount of capital that makes the insurance company to be solvent at least $\alpha \%$ of the time. TVaR is a conditional tail expectation and the TVaR at a confidence level $\alpha, \operatorname{TVaR}_{\alpha}(X)$, evaluates the expected loss amount incurred among the worst $(1-\alpha) \%$ scenarios.

Definition 3. For a random variable $X, \operatorname{TrTVaR}$ is defined as: 


$$
\begin{aligned}
\operatorname{TrTVaR}_{\alpha_{1}, \alpha_{2}}(X) & =\frac{1}{\alpha_{2}-\alpha_{1}} \int_{\alpha_{1}}^{\alpha_{2}} \operatorname{VaR}_{s}(X) \mathrm{d} s \\
& =\frac{\left(1-\alpha_{1}\right) \operatorname{TVaR}_{\alpha_{1}}(X)-\left(1-\alpha_{2}\right) \operatorname{TVaR}_{\alpha_{2}}(X)}{\alpha_{2}-\alpha_{1}},
\end{aligned}
$$

for some $0<\alpha_{1}<\alpha_{2}<1$.

It can easily be seen that TrTVaR is simply the average of VaR levels across a range of loss probabilities, this measure incorporates both the $\mathrm{VaR}$ and the TVaR risk measure. TrTVaR risk measure recovers TVaR risk measure when $\alpha_{1}=0$, $\alpha_{2}=1$. It is well known that the TVaR is a convex risk measure, while the VaR is not convex. The TrTVaR represents a compromise between the VaR and the TVaR, in the sense that only a part of the tail behaviour is measured by it, and as expected, it has similar properties to the VaR.

Property. 1) Monotonicity: $\operatorname{TrTVaR}_{\alpha_{1}, \alpha_{2}}(X) \leq \operatorname{Tr} T V a R_{\alpha_{1}, \alpha_{2}}(Y)$ provided that

$$
P\{X \leq Y\}=1 \text {; }
$$

2) Positive homogeneity: for any positive constant $c \geq 0$ and loss $X$,

$$
\operatorname{TrTVaR} \alpha_{\alpha_{1}, \alpha_{2}}(c X)=c \operatorname{TrTVaR} \alpha_{\alpha_{1}, \alpha_{2}}(X)
$$

3) Translation invariance: if $c$ is a constant, then:

$$
\operatorname{TrTVaR}_{\alpha_{1}, \alpha_{2}}(X+c)=\operatorname{TrTVaR}_{\alpha_{1}, \alpha_{2}}(X)+c ;
$$

4) Additivity for comonotonic risks: for any comonotonic random variables $X$ and $Y, \operatorname{TrTVaR}_{\alpha_{1}, \alpha_{2}}(X+Y)=\operatorname{TrTVaR}_{\alpha_{1}, \alpha_{2}}(X)+\operatorname{TrTVaR}_{\alpha_{1}, \alpha_{2}}(Y)$.

The robustness and efficiency properties depend on the choice for $0<\alpha_{1}<\alpha_{2}<1$ (the higher $\alpha_{2}$, the more efficient but also the less robust the estimator will be). In summary the TrTVaR is a non-convex and robust risk measure, and it has the advantage of being more tail sensitive than the VaR.

We now assume that the admissible set of ceded functions is given by:

$$
\mathbb{F}:=\{0 \leq R(x) \leq x \text { : both } R(x) \text { and } x-R(x) \text { are increasing functions }\} .
$$

This means that the reinsurer's payments to the insurer cannot exceed the insurer's losses, and that the ceded and retained loss functions are non-decreasing. These conditions are required to reduce moral hazards in reinsurance transactions. It was shown in (Chi \& Tan, 2011) that all functions $R \in \mathbb{F}$ are Lipschitz continuous and consequently differentiable almost everywhere.

In this paper, we study the Pareto-optimal reinsurance taking into account the interests of the insurer and the reinsurer, which means that neither of the two parties can be better off without making the other worse off. It is easy to see that a Pareto-optimal reinsurance contract exists if there is a contract that minimizes the convex combination of the objective functional of the insurer and the reinsurer. Indeed, the following proposition gives a sufficient condition for a reinsurance contract to be Pareto-optimal.

Proposition 1. All Pareto-optimal reinsurance policies $R$ in $\mathbb{F}$ can be determined by solving the problem: 


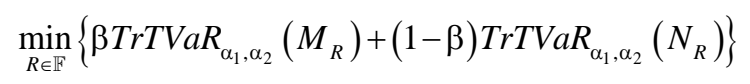

for some $\beta \in(0,1)$.

Proof. Let $R^{*} \in \arg \min \left\{\beta \operatorname{TrTVaR} \alpha_{\alpha_{1}, \alpha_{2}}\left(M_{R}\right)+(1-\beta) \operatorname{TrTVaR}_{\alpha_{1}, \alpha_{2}}\left(N_{R}\right)\right\}$ for some $\beta \in(0,1)$. If $R^{*} \stackrel{R \in \mathbb{P}}{\text { is }}$ not Pareto-optimal, then there exists a function $R$ in $\mathbb{F}$ such that $\operatorname{TrTVaR}_{\alpha_{1}, \alpha_{2}}\left(M_{R}\right) \leq \operatorname{TrTVaR}_{\alpha_{1}, \alpha_{2}}\left(M_{R^{*}}\right)$ and

$\operatorname{TrTVaR}_{\alpha_{1}, \alpha_{2}}\left(N_{R}\right) \leq \operatorname{TrTVaR}{\alpha_{1}, \alpha_{2}}_{2}\left(N_{R^{*}}\right)$, and at least one of the two inequalities is strict. Then:

$$
\begin{aligned}
& \beta \operatorname{TrTVaR}_{\alpha_{1}, \alpha_{2}}\left(M_{R}\right)+(1-\beta) \operatorname{TrTVaR} \alpha_{\alpha_{1}, \alpha_{2}}\left(N_{R}\right) \\
& <\beta \operatorname{TrTVaR}_{\alpha_{1}, \alpha_{2}}\left(M_{R^{*}}\right)+(1-\beta) \operatorname{TrTVaR}_{\alpha_{1}, \alpha_{2}}\left(N_{R^{*}}\right) .
\end{aligned}
$$

This is a contradiction to the assumed property of function $R^{*}$. Therefore, $R^{*}$ is the Pareto-optimal reinsurance policies. This completes the proof.

In this paper, we assume the premium principle is calculated by the expected value premium principle and TVaR premium principle, respectively. In view of Proposition 1, throughout the rest of this paper, we only need to determine optimal reinsurance policies by solving the optimisation problem:

$$
\min _{R \in \mathbb{F}}\left\{\beta \operatorname{TrTVaR} \alpha_{\alpha_{1}, \alpha_{2}}\left(M_{R}\right)+(1-\beta) \operatorname{TrTVaR} \alpha_{\alpha_{1}, \alpha_{2}}\left(N_{R}\right)\right\} .
$$

According to the additivity for comonotonic risks and translation invariance of TrTVaR, we obtain:

$$
\operatorname{TrTVaR}_{\alpha_{1}, \alpha_{2}}\left(N_{R}\right)=\operatorname{TrTVaR}_{\alpha_{1}, \alpha_{2}}(R(X))-P_{R},
$$

since the ceded loss $R(X)$ and the retained loss $X-R(X)$ are comonotonic, then:

$$
\operatorname{TrTVaR}_{\alpha_{1}, \alpha_{2}}\left(M_{R}\right)=\operatorname{TrTVaR}_{\alpha_{1}, \alpha_{2}}(X)-\operatorname{TrTVaR}_{\alpha_{1}, \alpha_{2}}(R(X))+P_{R} .
$$

Therefore, the optimization goal becomes:

$$
\min _{R \in \mathbb{F}}\left\{(1-2 \beta) \operatorname{Tr} T V a R_{\alpha_{1}, \alpha_{2}}(R(X))+(2 \beta-1) P_{R}\right\} .
$$

\section{Pareto-0ptimal Reinsurance Strategy}

\subsection{Pareto-Optimal Reinsurance Policies under Expected Value Principle}

In this section, we first study pareto-optimal reinsurance strategy when the premium principle is the expected value premium principle: $(1+\rho) E(R(x))$, where $\rho>0$ is the safety loading, the expected value premium principle recovers the net premium principle when $\rho=0$. In this setting, our optimal reinsurance problem becomes:

$$
\min _{R \in \mathbb{F}}\left\{(1-2 \beta) \operatorname{TrTVaR} \alpha_{\alpha_{1}, \alpha_{2}}(R(X))+(2 \beta-1)(1+\rho) E(R(X))\right\},
$$

that is:

$$
\min _{R \in \mathbb{F}}\left\{\frac{1-2 \beta}{\alpha_{2}-\alpha_{1}} \int_{\alpha_{1}}^{\alpha_{2}} R\left(\operatorname{VaR}_{s}(X)\right) \mathrm{d} s+(2 \beta-1)(1+\rho) \int_{0}^{1} R\left(\operatorname{VaR}_{s}(X)\right) \mathrm{d} s\right\} .
$$


Since the optimal reinsurance problem is always 0 when $\beta=\frac{1}{2}$, we only consider the case of $\beta>\frac{1}{2}$ and $\beta<\frac{1}{2}$. To solve the optimization problem, we quote proposition 1 in (Asimit, Badescu, \& Verdonck, 2013), and we now present it as lemma 1.

Lemma 1. Let $f(\cdot)$ be a real-valued function defined on $\left[s_{1}, s_{2}\right]$ with $0 \leq s_{1} \leq s_{2} \leq 1$. Then, $\min _{R \in \mathbb{F}} \int_{s_{1}}^{s_{2}} f(s) R\left(\operatorname{VaR}_{s}(X)\right) \mathrm{d} s$, subject to

$$
\begin{aligned}
& R\left(\operatorname{VaR}_{s_{1}}(X)\right)=\xi_{1}, \quad R\left(\operatorname{VaR}_{s_{2}}(X)\right)=\xi_{2} \text { is uniquely solved by: } \\
& R^{*}\left(X ; \xi_{1}, \xi_{2}\right) \\
& \quad=\left\{\begin{array}{l}
\left(X-\operatorname{VaR}_{s_{1}}(X)+\xi_{1}\right) \wedge \xi_{2}, \text { if } f(s)<0 \text { for all } s_{1} \leq s \leq s_{2}, \\
\xi_{1}+\left(X-\operatorname{VaR}_{s_{2}}(X)+\xi_{2}-\xi_{1}\right)_{+}, \text {if } f(s)>0 \text { for all } s_{1} \leq s \leq s_{2},
\end{array}\right.
\end{aligned}
$$

where $\left(\xi_{1}, \xi_{2}\right)$ are some constants such that $0 \leq \xi_{2}-\xi_{1} \leq \operatorname{VaR}_{s_{2}}(X)-\operatorname{VaR}_{s_{1}}(X)$.

For simplicity, we define the following notations:

$$
\begin{aligned}
& \xi_{1}=R\left(\operatorname{VaR}_{\alpha_{1}}(X)\right) \\
& \xi_{2}=R\left(\operatorname{VaR}_{\alpha_{2}}(X)\right) \\
& \rho^{*}=\frac{\rho}{1+\rho} \\
& n=1-\frac{1-\alpha_{2}}{1-\left(\alpha_{2}-\alpha_{1}\right)(1+\rho)} \\
& t=1-\frac{1}{\left(\alpha_{2}-\alpha_{1}\right)(1+\rho)}
\end{aligned}
$$

Theorem 1. Under the condition $\frac{1}{2}<\beta<1$, the Pareto-optimal reinsurance policies are given as follows:

1) If $1+\rho<\frac{1}{\alpha_{2}-\alpha_{1}}$, then:

$$
R^{*}(x)= \begin{cases}\left(x-\operatorname{VaR}_{\rho^{*}}(X)\right)_{+} \wedge\left(\operatorname{VaR}_{n}(X)-\operatorname{VaR}_{\rho_{*}^{*}}(X)\right)_{+}, & \text {if }(1+\rho)\left(1-\alpha_{1}\right)>1, \\ \left(x-\operatorname{VaR}_{t}(X)\right)_{+} \wedge\left(\operatorname{VaR}_{\alpha_{1}}(X)-\operatorname{VaR}_{t}(X)\right)_{+}, & \text {if }(1+\rho)\left(1-\alpha_{1}\right) \leq 1 .\end{cases}
$$

2) If $1+\rho>\frac{1}{\alpha_{2}-\alpha_{1}}$, then:

$R^{*}(x)=\left\{\begin{array}{l}\left(x-\operatorname{VaR}_{\rho^{*}}(X)\right)_{+} I_{\left\{0<x \leq \operatorname{VaR}_{\alpha_{1}}(X)\right\}}+\left(\operatorname{VaR}_{\alpha_{1}}(X)-\operatorname{VaR}_{\rho^{*}}(X)\right)_{+} I_{\left\{x>V a R_{\alpha_{1}}(X)\right\}}, \\ \quad \text { if }(1+\rho)\left(1-\alpha_{1}\right)<1, \\ 0, \quad \text { if }(1+\rho)\left(1-\alpha_{1}\right) \geq 1 .\end{array}\right.$

3) If $1+\rho=\frac{1}{\alpha_{2}-\alpha_{1}}$, then:

$$
R^{*}(x)=R(x) I_{\left\{\operatorname{VaR}_{\alpha_{1}}(x)<x \leq \operatorname{VaR}_{\alpha_{2}}(x)\right\}},
$$


where $R^{*}(x)$ is any increasing Lipschitz continuous function such that $R^{*}(x) \in \mathbb{F}$.

Proof. The equivalent form of (4) is:

$$
\begin{aligned}
& \min _{R \in \mathbb{F}}\left\{\left((2 \beta-1)(1+\rho)+\frac{1-2 \beta}{\alpha_{2}-\alpha_{1}}\right) \int_{\alpha_{1}}^{\alpha_{2}} R\left(\operatorname{VaR}_{s}(X)\right) \mathrm{d} s\right. \\
& \left.+m\left(\int_{0}^{\alpha_{1}}+\int_{\alpha_{2}}^{1}\right) R\left(\operatorname{VaR}_{s}(X)\right) \mathrm{d} s\right\} .
\end{aligned}
$$

1) If $1+\rho<\frac{1}{\alpha_{2}-\alpha_{1}}$, by Lemma 1 , we get that (5) is solved by:

$$
R^{*}\left(x ; \xi_{1}, \xi_{2}\right)=\left(x-\operatorname{VaR}_{\alpha_{1}}(X)+\xi_{1}\right)_{+} \wedge \xi_{2},
$$

where $\left(\xi_{1}, \xi_{2}\right) \in \mathbb{C}_{1}$ is a vector of constants with:

$$
\begin{aligned}
\mathbb{C}_{1}= & \left\{\left(\xi_{1}, \xi_{2}\right): 0 \leq \xi_{1} \leq \operatorname{VaR}_{\alpha_{1}}(X) ; 0 \leq \xi_{2} \leq \operatorname{VaR}_{\alpha_{2}}(X) ;\right. \\
& \left.0 \leq \xi_{2}-\xi_{1} \leq \operatorname{VaR}_{\alpha_{2}}(X)-\operatorname{VaR}_{\alpha_{1}}(X)\right\} .
\end{aligned}
$$

Thus, the second step is to minimize:

$$
\begin{aligned}
H_{1}\left(\xi_{1}, \xi_{2}\right)= & \frac{1-2 \beta}{\alpha_{2}-\alpha_{1}}\left(\xi_{1}\left(1-\alpha_{1}\right)-\xi_{2}\left(1-\alpha_{2}\right)+\int_{\operatorname{VaR}_{\alpha_{1}}(X)}^{\operatorname{VaR}_{\alpha_{1}}(X)+\xi_{2}-\xi_{1}} S_{X}(x) \mathrm{d} x\right) \\
& +(2 \beta-1)(1+\rho) \int_{\operatorname{VaR}_{\alpha_{1}}(x)-\xi_{1}}^{\operatorname{VaR}_{\alpha_{1}}(x)+\xi_{2}-\xi_{1}} S_{X}(x) \mathrm{d} x .
\end{aligned}
$$

Note that the derivative:

$$
\begin{aligned}
\frac{\partial H_{1}}{\partial \xi_{2}}= & \left(\frac{1-2 \beta}{\alpha_{2}-\alpha_{1}}+(2 \beta-1)(1+\rho)\right) S_{X}\left(\operatorname{VaR}_{\alpha_{1}}(X)+\xi_{2}-\xi_{1}\right) \\
& +\frac{(2 \beta-1)\left(1-\alpha_{2}\right)}{\alpha_{2}-\alpha_{1}}
\end{aligned}
$$

is increasing in $\left[\xi_{1}, \xi_{1}+\operatorname{VaR}_{\alpha_{2}}(X)-\operatorname{VaR}_{\alpha_{1}}(X)\right]$ with respect to $\xi_{2}$.

a) When $(1+\rho)\left(1-\alpha_{1}\right)>1$, the derivative $\left.\frac{\partial H_{1}}{\partial \xi_{2}}\right|_{\xi_{2}=\xi_{1}}<0$. Since $m>0$, we obtain $\left.\frac{\partial H_{1}}{\partial \xi_{2}}\right|_{\xi_{2}=\xi_{1}+V_{\alpha_{\alpha 2}}(X)-\operatorname{VaR}_{\alpha_{1}}(X)}>0$. Therefore, $H_{1}$ attains its minimum value at $\xi_{2}^{*}=\operatorname{VaR}_{s(\beta)}(X)-\operatorname{VaR}_{\alpha_{1}}(X)+\xi_{1}$. Now,

$$
\begin{aligned}
H_{1}\left(\xi_{1}, \xi_{2}^{*}\right)= & \frac{1-2 \beta}{\alpha_{2}-\alpha_{1}}\left(\xi_{1}\left(1-\alpha_{1}\right)-\left(\operatorname{VaR}_{s(\beta)}(X)-\operatorname{VaR}_{\alpha_{1}}(X)+\xi_{1}\right)\left(1-\alpha_{2}\right)\right. \\
& \left.+\int_{\operatorname{VaR}_{\alpha_{1}}(X)}^{\operatorname{VaR}(X)} S_{X}(x) \mathrm{d} x\right)+(2 \beta-1)(1+\rho) \int_{\operatorname{VaR}_{\alpha_{1}}(X)-\xi_{1}}^{\operatorname{VaR}_{n}(X)} S_{X}(x) \mathrm{d} x .
\end{aligned}
$$

It is easy to see, the derivative $\frac{\partial H_{1}}{\partial \xi_{1}}=(1-2 \beta)+(2 \beta-1)(1+\rho) S_{X}\left(\operatorname{VaR}_{\alpha_{1}}(X)-\xi_{1}\right)$ is negative if and only if $F\left(\operatorname{VaR}_{\alpha_{1}}(X)-\xi_{1}\right)>\rho^{*}$, which is equivalent to $\xi_{1}<\operatorname{VaR}_{\alpha_{1}}(X)-\operatorname{VaR}_{\rho^{*}}(X)$. Therefore, $H_{1}$ attains its minimum value at $\xi_{1}^{*}=\operatorname{VaR}_{\alpha_{1}}(X)-\operatorname{VaR}_{\rho^{*}}(X)$. 
b) When $(1+\rho)\left(1-\alpha_{1}\right) \leq 1$, we have $\left.\frac{\partial H_{1}}{\partial \xi_{2}}\right|_{\xi_{2}=\xi_{1}} \geq 0$. Thus, $H_{1}$ attains its minimum value at $\xi_{2}^{*}=\xi_{1}$. Now,

$$
H_{1}\left(\xi_{1}, \xi_{2}^{*}\right)=\frac{1-2 \beta}{\alpha_{2}-\alpha_{1}} \xi_{1}+(2 \beta-1)(1+\rho) \int_{\operatorname{VaR}_{\alpha_{1}}(X)-\xi_{1}}^{V_{\alpha_{1}}(x)} S_{X}(x) \mathrm{d} x
$$

and:

$$
\frac{\partial H_{1}}{\partial \xi_{1}}=\frac{1-2 \beta}{\alpha_{2}-\alpha_{1}}+(2 \beta-1)(1+\rho) S_{X}\left(\operatorname{VaR}_{\alpha_{1}}(X)-\xi_{1}\right)
$$

thus $\frac{\partial H_{1}}{\partial \xi_{1}}$ is increasing in $\left[0, \operatorname{VaR}_{\alpha_{1}}(X)\right]$ with respect to $\xi_{1}$. Since $\left.\frac{\partial H_{1}}{\partial \xi_{1}}\right|_{\xi_{1}=0}<0$ and $\left.\frac{\partial H_{1}}{\partial \xi_{1}}\right|_{\xi_{1}=V a R_{\alpha_{1}}(X)} \geq 0$, it is easy to see that $H_{1}$ attains its minimum value at $\xi_{1}^{*}=\operatorname{VaR}_{\alpha_{1}}(X)$. Therefore, $R^{*}(x ; \xi)=x \wedge \operatorname{VaR}_{\alpha_{1}}(X)$;

When $\left.\frac{\partial H_{1}}{\partial \xi_{1}}\right|_{\xi_{1}=\operatorname{VaR}_{\alpha_{1}}(X)}>0, H_{1}$ attains its minimum value at $\xi_{1}^{*}=\operatorname{VaR}_{\alpha_{1}}(X)-\operatorname{VaR}_{t}(X)$. Therefore, $R^{*}(x)=\left(x-\operatorname{VaR}_{t}(X)\right)_{+} \wedge\left(\operatorname{VaR}_{\alpha_{1}}(X)-\operatorname{VaR}_{t}(X)\right)_{+}$. Note that $\operatorname{VaR}_{t}(X)=0$ when $\left.\frac{\partial H_{1}}{\partial \xi_{1}}\right|_{\xi_{1}=V_{a R_{\alpha_{1}}}(X)}=0$. Therefore, $R^{*}(x)=\left(x-\operatorname{VaR}_{t}(X)\right)_{+} \wedge\left(\operatorname{VaR}_{\alpha_{1}}(X)-\operatorname{VaR}_{t}(X)\right)_{+}$when $\left.\frac{\partial H_{1}}{\partial \xi_{1}}\right|_{\xi_{1}=V a R_{\alpha_{1}}(X)}=0$.

The proof for the remaining case is similar to the above, and thus we omit the proof.

Theorem 2. Under the condition $0<\beta<\frac{1}{2}$, the Pareto-optimal reinsurance policies are given as follows:

1) If $1+\rho>\frac{1}{\alpha_{2}-\alpha_{1}}$, then: $R^{*}(x)=\left\{\begin{array}{l}\left(x \wedge \operatorname{VaR}_{\rho^{*}}(X)\right) I_{\left\{0<x \leq \operatorname{VaR}_{\alpha_{2}}(X)\right\}}+\left(x-\operatorname{VaR}_{\alpha_{2}}(X)+\operatorname{VaR}_{\rho^{*}}(X)\right) I_{\left\{x>\operatorname{VaR}_{\alpha_{2}}(X)\right\}}, \\ \quad \text { if }(1+\rho)\left(1-\alpha_{1}\right)<1, \\ \left(x \wedge \operatorname{VaR}_{\alpha_{1}}(X)\right) I_{\left\{0<x \leq \operatorname{VaR}_{\alpha_{2}}(X)\right\}}+\left(x-\operatorname{VaR}_{\alpha_{2}}(X)+\operatorname{VaR}_{\alpha_{1}}(X)\right) I_{\left\{x>\operatorname{VaR}_{\alpha_{2}}(X)\right\}}, \\ \quad \text { if }(1+\rho)\left(1-\alpha_{1}\right) \geq 1 .\end{array}\right.$ 2) If $1+\rho<\frac{1}{\alpha_{2}-\alpha_{1}}$, then:

$$
R^{*}(x)=\left\{\begin{aligned}
\left(x \wedge \operatorname{VaR}_{\rho^{*}}(X)\right) I_{\left\{0<x \leq \operatorname{VaR}_{\alpha_{1}}(x)\right\}}+\left(x+\operatorname{VaR}_{\rho^{*}}(X)-\operatorname{VaR}_{\alpha_{1}}(X)\right) I_{\left\{x>\operatorname{VaR}_{\alpha_{1}}(X)\right\}}, \\
\quad \text { if }(1+\rho)\left(1-\alpha_{1}\right)<1, \\
x, \quad \text { if }(1+\rho)\left(1-\alpha_{1}\right) \geq 1 .
\end{aligned}\right.
$$


3) If $1+\rho=\frac{1}{\alpha_{2}-\alpha_{1}}$, then:

$$
R^{*}(x)=x I_{\left\{0<x \leq V a R_{\alpha_{1}}(x) \text { or } x>\operatorname{VaR}_{\alpha_{2}}(x)\right\}}+R(x) I_{\left\{\operatorname{VaR}_{\alpha_{1}}(x)<x \leq \operatorname{VaR}_{\alpha_{2}}(x)\right\}},
$$

where $R^{*}(x)$ is any increasing Lipschitz continuous function such that $R^{*}(x) \in \mathbb{F}$.

Proof. The proofs of theorem 2 are omitted, since they are quite similar to those of Theorem 1 .

\subsection{Pareto-Optimal Reinsurance Policies under TVaR Principle}

In this section, we assume the premium is calculated by TVaR premium principle: $(1+\theta) T \operatorname{VaR}_{\alpha}(R(X))$, where $\theta \in[0,1]$ is the safety loading. In this setting, our optimal reinsurance problem becomes:

$$
\min _{R \in \mathbb{F}}\left\{(1-2 \beta) \operatorname{TrTVaR}_{\alpha_{1}, \alpha_{2}}(R(x))+(2 \beta-1)(1+\theta) \operatorname{TVaR}_{\alpha}(R(x))\right\} .
$$

From the mathematical point of view, the confidence level $\alpha$ can be larger than confidence levels $\alpha_{1}$. However, $\alpha$ is usually smaller while $\alpha_{1}$ are usually larger in practice. So, we assume further $\alpha<\alpha_{1}$ to avoid complex and lengthy discussions in this section. Since the target function is always 0 when $\beta=\frac{1}{2}$, we only consider the case of $\beta>\frac{1}{2}$ and $\beta<\frac{1}{2}$.

For simplicity, we define the following notations:

$$
\begin{aligned}
& \xi=R\left(\operatorname{VaR}_{\alpha}(X)\right) \\
& \xi_{1}=R\left(\operatorname{VaR}_{\alpha_{1}}(X)\right) \\
& \xi_{2}=R\left(\operatorname{VaR}_{\alpha_{2}}(X)\right) \\
& m_{0}=\frac{(2 \beta-1)(1+\theta)}{1-\alpha} \\
& u=1-\frac{\left(1-\alpha_{2}\right)(1-\alpha)}{(1-\alpha)-(1+\theta)\left(\alpha_{2}-\alpha_{1}\right)} \\
& v=1-\frac{1-\alpha}{1+\theta}
\end{aligned}
$$

Theorem 3. Under the condition $\frac{1}{2}<\beta<1$, the Pareto-optimal reinsurance policies are given as follows:

1) If $0<m_{0}<\frac{2 \beta-1}{\alpha_{2}-\alpha_{1}}$, then:

$$
R^{*}(x)=\left\{\begin{aligned}
\left(x-\operatorname{VaR}_{v}(X)\right)_{+} \wedge\left(\operatorname{VaR}_{u}(X)-\operatorname{VaR}_{v}(X)\right)_{+}, \\
\quad \text { if }(1+\theta)\left(1-\alpha_{1}\right)<1-\alpha, \\
0, \quad \text { if }(1+\theta)\left(1-\alpha_{1}\right) \geq 1-\alpha .
\end{aligned}\right.
$$

2) If $m_{0}>\frac{2 \beta-1}{\alpha_{2}-\alpha_{1}}$, then $R^{*}(x)=0$.

3) If $m_{0}=\frac{2 \beta-1}{\alpha_{2}-\alpha_{1}}$, then $R^{*}(x)=R(x) I_{\left\{\operatorname{VaR}_{\alpha_{1}}(x)<x<\operatorname{VaR}_{\alpha_{2}}(x)\right\}}$, where $R^{*}(x)$ is 
any increasing Lipschitz continuous function such that $R^{*}(x) \in \mathbb{F}$.

Proof. The equivalent form of (6) is:

$$
\min _{R \in \mathbb{F}}\left\{m_{0}\left(\int_{\alpha}^{\alpha_{1}}+\int_{\alpha_{2}}^{1}\right) R\left(\operatorname{VaR}_{s}(X)\right) \mathrm{d} s+\left(m_{0}-\frac{2 \beta-1}{\alpha_{2}-\alpha_{1}}\right) \int_{\alpha_{1}}^{\alpha_{2}} R\left(\operatorname{VaR}_{s}(X)\right) \mathrm{d} s\right\} .
$$

1) If $0<m_{0}<\frac{2 \beta-1}{\alpha_{2}-\alpha_{1}}$, by Lemma 1 , we get that (7) is solved by:

$$
\begin{aligned}
& R^{*}\left(x ; \xi_{,} \xi_{1}, \xi_{2}\right) \\
& =\left\{\begin{array}{lc}
\xi+\left(x-\operatorname{VaR}_{\alpha_{1}}(X)+\xi_{1}-\xi\right)_{+}, & \operatorname{VaR}_{\alpha}(X) \leq x \leq \operatorname{VaR}_{\alpha_{1}}(X), \\
\left(x-\operatorname{VaR}_{\alpha_{1}}(X)+\xi_{1}\right) \wedge \xi_{2}, & \operatorname{VaR}_{\alpha_{1}}(X)<x \leq \operatorname{VaR}_{\alpha_{2}}(X), \\
\xi_{2}, & x>\operatorname{VaR}_{\alpha_{2}}(X),
\end{array}\right.
\end{aligned}
$$

where $\left(\xi, \xi_{1}, \xi_{2}\right) \in \mathbb{C}_{2}$ is a vector of constants with:

$$
\begin{aligned}
\mathbb{C}_{2}=\{( & \left(\xi, \xi_{1}, \xi_{2}\right): 0 \leq \xi \leq \operatorname{VaR}_{\alpha}(X) ; 0 \leq \xi_{1} \leq \operatorname{VaR}_{\alpha_{1}}(X) \\
& 0 \leq \xi_{2} \leq \operatorname{VaR}_{\alpha_{2}}(X) ; 0 \leq \xi_{2}-\xi_{1} \leq \operatorname{VaR}_{\alpha_{2}}(X)-\operatorname{VaR}_{\alpha_{1}}(X) \\
& 0 \leq \xi_{1}-\xi \leq \operatorname{VaR}_{\alpha_{1}}(X)-\operatorname{VaR}_{\alpha}(X) \\
& \left.0 \leq \xi_{2}-\xi \leq \operatorname{VaR}_{\alpha_{2}}(X)-\operatorname{VaR}_{\alpha}(X)\right\}
\end{aligned}
$$

Thus, the second step is to minimize:

$$
\begin{aligned}
H_{2}\left(\xi, \xi_{1}, \xi_{2}\right)= & \frac{1-2 \beta}{\alpha_{2}-\alpha_{1}}\left(\xi_{1}\left(1-\alpha_{1}\right)-\xi_{2}\left(1-\alpha_{2}\right)+\int_{\operatorname{VaR}_{\alpha_{1}}(X)}^{V_{\alpha_{1}}(X)+\xi_{2}-\xi_{1}} S_{X}(x) \mathrm{d} x\right) \\
& +\frac{(2 \beta-1)(1+\theta)}{1-\alpha}\left(\xi(1-\alpha)+\int_{\operatorname{VaR}_{\alpha_{1}}(X)+\xi-\xi_{1}}^{V_{\alpha_{\alpha_{1}}}(X)+\xi_{2}-\xi_{1}} S_{X}(x) \mathrm{d} x\right) .
\end{aligned}
$$

The derivative:

$$
\frac{\partial H_{2}}{\partial \xi_{2}}=\left(m_{0}-\frac{2 \beta-1}{\alpha_{2}-\alpha_{1}}\right) S_{X}\left(\operatorname{VaR}_{\alpha_{1}}(X)+\xi_{2}-\xi_{1}\right)+\frac{(2 \beta-1)\left(1-\alpha_{2}\right)}{\alpha_{2}-\alpha_{1}}
$$

is increasing in $\left[\xi_{1}, \xi_{1}+\operatorname{VaR}_{\alpha_{2}}(X)-\operatorname{VaR}_{\alpha_{1}}(X)\right]$ with respect to $\xi_{2}$.

a) When $(1+\theta)\left(1-\alpha_{1}\right)<1-\alpha$, we have $\left.\frac{\partial H_{2}}{\partial \xi_{2}}\right|_{\xi_{2}=\xi_{1}}<0$ and $\left.\frac{\partial H_{2}}{\partial \xi_{2}}\right|_{\xi_{2}=\xi_{1}+\operatorname{VaR}_{\alpha_{2}}(X)-\operatorname{VaR}_{\alpha_{1}}(X)}>0$. Thus, $H_{2}$ attains the minimum value at $\xi_{2}^{*}=\operatorname{VaR}_{u}(X)-\operatorname{VaR}_{\alpha_{1}}(X)+\xi_{1}$. Now,

$$
\begin{aligned}
H_{2}\left(\xi, \xi_{1}, \xi_{2}^{*}\right)= & \frac{1-2 \beta}{\alpha_{2}-\alpha_{1}}\left(\xi_{1}\left(1-\alpha_{1}\right)-\left(\operatorname{VaR}_{u}(X)-\operatorname{VaR}_{\alpha_{1}}(X)+\xi_{1}\right)\left(1-\alpha_{2}\right)\right. \\
& \left.+\int_{\operatorname{VaR}_{\alpha_{1}}(X)}^{\operatorname{VaR}_{u}(X)} S_{X}(x) \mathrm{d} x\right) \\
& +\frac{(2 \beta-1)(1+\theta)}{1-\alpha}\left(\xi(1-\alpha)+\int_{\operatorname{VaR}_{\alpha_{1}}(X)+\xi-\xi_{1}}^{\operatorname{VaR}_{u}(X)} S_{X}(x) \mathrm{d} x\right)
\end{aligned}
$$

the derivative $\frac{\partial H_{2}}{\partial \xi_{1}}=(1-2 \beta)+\frac{(2 \beta-1)(1+\theta)}{1-\alpha} S_{X}\left(\operatorname{VaR}_{\alpha_{1}}(X)+\xi-\xi_{1}\right)$ is increas- 
ing in $\left[\xi, \xi+\operatorname{VaR}_{\alpha_{1}}(X)-\operatorname{VaR}_{\alpha}(X)\right]$. It is easy to see $\left.\frac{\partial H_{2}}{\partial \xi_{1}}\right|_{\xi_{1}=\xi}<0$ and $\left.\frac{\partial H_{2}}{\partial \xi_{1}}\right|_{\xi_{1}=\xi+\operatorname{VaR}_{\alpha_{1}}(X)-\operatorname{VaR}_{\alpha}(X)}>0$, then $H_{2}$ attains its minimum value at $\xi_{1}^{*}=\operatorname{VaR}_{\alpha_{1}}(X)-\operatorname{VaR}_{v}(X)+\xi$. Now,

$$
\begin{aligned}
H_{2}\left(\xi, \xi_{1}^{*}, \xi_{2}^{*}\right)= & \frac{1-2 \beta}{\alpha_{2}-\alpha_{1}}\left(\left(\operatorname{VaR}_{\alpha_{1}}(X)-\operatorname{VaR}_{v}(X)+\xi\right)\left(1-\alpha_{1}\right)\right. \\
& \left.-\left(\operatorname{VaR}_{u}(X)-\operatorname{VaR}_{v}(X)+\xi\right)\left(1-\alpha_{2}\right)+\int_{\operatorname{VaR}_{\alpha_{1}}(X)}^{\operatorname{VaR}_{u}(X)} S_{X}(x) \mathrm{d} x\right) \\
& +\frac{(2 \beta-1)(1+\theta)}{1-\alpha}\left(\xi(1-\alpha)+\int_{\operatorname{VaR}_{v}(X)}^{\operatorname{VaR}_{u}(X)} S_{X}(x) \mathrm{d} x\right) .
\end{aligned}
$$

It is easy to see $\frac{\partial H_{2}}{\partial \xi_{1}}=\theta(2 \beta-1)>0$, then $H_{2}$ attains its minimum value at $\xi^{*}=0$. Therefore, $R^{*}(x)=\left(x-\operatorname{VaR}_{v}(X)\right)_{+} \wedge\left(\operatorname{VaR}_{u}(X)-\operatorname{VaR}_{v}(X)\right)_{+}$.

b) Since $(1+\theta)\left(1-\alpha_{1}\right) \geq 1$, we have $\left.\frac{\partial H_{2}}{\partial \xi_{2}}\right|_{\xi_{2}=\xi_{1}} \geq 0$. Therefore, $H_{2}$ attains its minimum value at $\xi_{2}^{*}=\xi_{1}$. Now,

$$
\begin{aligned}
& H_{2}\left(\xi, \xi_{1}, \xi_{2}^{*}\right) \\
& =(1-2 \beta) \xi_{1}+\frac{(2 \beta-1)(1+\theta)}{1-\alpha}\left(\xi(1-\alpha)+\int_{\operatorname{VaR}_{\alpha_{1}}(X)+\xi_{2}-\xi_{1}}^{V a R_{\alpha_{1}}(X)} S_{X}(x) \mathrm{d} x\right)
\end{aligned}
$$

and $\frac{\partial H_{2}}{\partial \xi_{1}}=(1-2 \beta)+\frac{(2 \beta-1)(1+\theta)}{1-\alpha} S_{X}\left(\operatorname{VaR}_{\alpha_{1}}(X)+\xi-\xi_{1}\right)$, the derivative $\frac{\partial H_{2}}{\partial \xi_{1}}$ is increasing in $\left[\xi, \xi+\operatorname{VaR}_{\alpha_{1}}(X)-\operatorname{VaR}_{\alpha}(X)\right]$ with respect to $\xi_{1}$. It is easy to see, $\left.\frac{\partial H_{1}}{\partial \xi_{1}}\right|_{\xi_{1}=\xi}>0$, then, $H_{2}$ attains its minimum value at $\xi_{1}^{*}=\xi$. Now, $H_{1}\left(\xi, \xi_{1}^{*}, \xi_{2}^{*}\right)=(1-2 \beta) \xi+\frac{(2 \beta-1)(1+\theta)}{1-\alpha} \xi$. Since $\frac{\partial H_{2}}{\partial \xi}=\theta(2 \beta-1)>0$, then $H_{2}$ attains the minimum value at $\xi=0$. Therefore, $R^{*}(x)=0$.

The proof for the remaining cases is similar to the above, and thus we omit the proof.

Theorem 4. Under the condition $0<\beta<\frac{1}{2}$, the Pareto-optimal reinsurance policies are given as follows:

1) If $m_{0}<\frac{2 \beta-1}{\alpha_{2}-\alpha_{1}}$, then:

$$
R^{*}(x)=\left\{\begin{aligned}
\left(x \wedge \operatorname{VaR}_{w}(X)\right) I_{\left\{\operatorname{VaR}_{\alpha}(X)<x<\operatorname{VaR}_{\alpha_{1}}(X)\right\}}+\left(x+\operatorname{VaR}_{w}(X)-\operatorname{VaR}_{\alpha_{1}}(X)\right) I_{\left\{x>\operatorname{VaR}_{\alpha_{1}}(X)\right\}}, \\
\quad \text { if }(1+\theta)\left(1-\alpha_{1}\right)<1-\alpha, \\
x, \quad \text { if }(1+\theta)\left(1-\alpha_{1}\right) \geq 1-\alpha .
\end{aligned}\right.
$$

2) If $\frac{2 \beta-1}{\alpha_{2}-\alpha_{1}}<m_{0}<0$, then: 


$$
\begin{aligned}
& R^{*}(x)=\left\{\begin{aligned}
\left(x \wedge \operatorname{VaR}_{p}(X)\right) I_{\left\{\operatorname{VaR}_{\alpha}(X)<x<\operatorname{VaR}_{\alpha_{1}}(X)\right\}}+\left(x+\operatorname{VaR}_{p}(X)-\operatorname{VaR}_{z}(X)\right) I_{\left\{x>\operatorname{VaR}_{\alpha_{1}}(X)\right\}}, \\
\quad \text { if }(1+\theta)\left(1-\alpha_{1}\right)<1-\alpha, \\
x, \quad \text { if }(1+\theta)\left(1-\alpha_{1}\right) \geq 1-\alpha .
\end{aligned}\right. \\
& \text { 3) If } m_{0}=\frac{2 \beta-1}{\alpha_{2}-\alpha_{1}}, \text { then: } \\
& R^{*}(x)=x I_{\left\{\operatorname{VaR}_{\alpha}(X)<x \leq \operatorname{VaR}_{\alpha_{1}}(X), x>\operatorname{VaR}_{\alpha_{2}}(X)\right\}}+R(x) I_{\left\{\operatorname{VaR}_{\alpha_{1}}(X)<x \leq \operatorname{VaR}_{\alpha_{2}}(X)\right\}},
\end{aligned}
$$

where $R^{*}(x)$ is any increasing Lipschitz continuous function such that $R^{*}(x) \in \mathbb{F}$.

Proof. The proofs of theorem 4 are omitted, since they are quite similar to those of Theorem 3.

\section{Conclusion}

In this paper, we explore optimal reinsurance contracts which take into account both the insurer's aims and the reinsurer's goals. The models and problems proposed in this paper are interested in theory and applications: reinsurance plays a vital role in insurance activities because of its advantages of dispersing risks, stabilizing operations and optimizing resource allocation. In practice, it is unreasonable for most of the reinsurance optimization problems to consider only the interests of one of the parties. Therefore, it is very meaningful and necessary to study the optimal reinsurance strategy which takes into account the interests of both insurers and reinsurers. In this paper, we determine Pareto-optimal reinsurance policies under which one party's risk cannot be reduced without increasing that of the other party in the reinsurance contract. When the losses of an insurer and a reinsurer are both measured by TrTVaR risk measures, we obtain the explicit forms of the Pareto-optimal reinsurance contracts under the expected value premium principle and TVaR premium principle, respectively. The conclusion in this paper will provide the theoretical basis for the insurer and the reinsurer to determine the reinsurance contract.

Limitations of this paper and future research direction: our model assumes that the insurer may transfer the risk to only one reinsurance company. More realistic situations involve multiple reinsurance risk transfers available on the market. It is likely that each reinsurer has its own pricing model, and the cedent may choose to transfer specific layers from the total risk to different reinsurers. Therefore, we also wish to point out that further research on this topic is needed. First, the research on one reinsurer can be extended to two or more reinsurers to establish the optimal solution. Second, risk measure can be extended to the more general measure of distorted risk measure. Third, the premium principle can be extended to more general premiums such as Wang's premium principle. We hope that these three important open problems can be solved in future research. 


\section{Acknowledgements}

The research was supported by Social Science Planning Project of Shandong Province (20CTJJ02).

\section{Conflicts of Interest}

The authors declare no conflicts of interest regarding the publication of this paper.

\section{References}

Arrow, K. J. (1963). Uncertainty and the Welfare Economics of Medical Care. The American Economic Review, 53, 941-973. https://www.jstor.org/stable/1812044?seq=1

Asimit, A. V., Badescu, A. M., \& Verdonck, T. (2013). Optimal Risk Transfer under Quantile-Based Risk Measurers. Insurance Mathematics and Economics, 53, 252-265. https://doi.org/10.1016/j.insmatheco.2013.05.005

Bernard, C., \& Tian, W. (2009). Optimal Reinsurance Arrangements under Tail Risk Measures. The Journal of Risk and Insurance, 76, 709-725. https://doi.org/10.1111/j.1539-6975.2009.01315.x

Borch, K. (1960). An Attempt to Determine the Optimum Amount of Stop Loss Reinsurance Reinsurance. Transactions of the 16th International Congress of Actuaries, 2, 597-610.

Cai, J., \& Tan, K. S. (2007). Optimal Retention for a Stop-Loss Reinsurance under the VaR and CTE Risk Measures. ASTIN Bulletin, 37, 93-112. https://doi.org/10.1017/S0515036100014756

Cai, J., Liu, H. Y., \& Wang, R. D. (2017). Pareto-Optimal Reinsurance Arrangements under General Model Settings. Insurance Mathematics and Economics, 77, 24-37. https://doi.org/10.1016/j.insmatheco.2017.08.004

Cai, J., Tan, K. S., Weng, C., \& Zhang, Y. (2008). Optimal Reinsurance under VaR and CTE Risk Measures. Insurance Mathematics and Economics, 43, 185-196. https://doi.org/10.1016/j.insmatheco.2008.05.011

Cheung, K. C. (2010). Optimal Reinsurance Revisited-A Geometric Approach. ASTIN Bulletin, 40, 221-239. https://doi.org/10.2143/AST.40.1.2049226

Cheung, K. C., Sung, K., Yam, S., \& Yung, S. (2014). Optimal Reinsurance under General Law-Invariant Risk Measures. Scandinavian Actuarial Journal, 1, 72-91. https://doi.org/10.1080/03461238.2011.636880

Chi, Y., \& Tan, K. (2013). Optimal Reinsurance with General Premium Principles. Insurance Mathematics and Economics, 52, 180-189. https://doi.org/10.1016/j.insmatheco.2012.12.001

Chi, Y., \& Tan, K. S. (2011). Optimal Reinsurance under VaR and CVaR Risk Measures: A Simplified Approach. ASTIN Bulletin, 41, 487-509.

Cont, R., Deguest, R., \& Scandalo, G. (2010). Robustness and Sensitivity Analysis of Risk Measurement Procedures. Quantitative Finance, 10, 593-606. https://doi.org/10.1080/14697681003685597

Fang, Y., Wang, X., Liu, H., \& Li, T. (2019). Pareto-Optimal Reinsurance for Both the Insurer and the Reinsurer with General Premium Principles. Communications in Statistics-Theory and Methods, 48, 6134-6154. https://doi.org/10.1080/03610926.2018.1528364

Jiang, W., Hong, H., \& Ren, J. (2018). On Pareto-Optimal Reinsurance with Constraints 
under Distortion Risk Measures. European Actuarial Journal, 8, 215-243. https://doi.org/10.1007/s13385-017-0163-1

Jiang, W., Ren, J., Zitikis, R., \& Tang, Q. (2017). Optimal Reinsurance Policies under the VaR Risk Measure When the Interests of Both the Cedent and the Reinsurer Are Taken into Account. Risks, 5, 1-22. https://doi.org/10.3390/risks5010011

Jiang, W. J., Hong, H. P., \& Ren, J. D. (2021). Pareto-Optimal Reinsurance Policies with Maximal Synergy. Insurance: Mathematics and Economics, 96, 185-198.

https://doi.org/10.1016/j.insmatheco.2020.11.009 